\title{
Temporal Evolution of Multipactor Discharge
}

\author{
R. Kishek, Y. Y. Lau, and R. M. Gilgenbach \\ Department of Nuclear Engineering \\ University of Michigan \\ Ann Arbor, MI 48109-2104
}

\begin{abstract}
Multipactor is an important resonant discharge phenomenon on which there have been surprisingly few publications in the open literature. Here, we extend the theoretical analysis of an idealized model. Emphases have been placed on the mutual interactions between the multipactor discharge and the rf. We show that the multipactor current may reach a very high level, transiently, before it settles to a steady state. The multipactor current saturates primarily by its loading of the cavity; the image space charge force associated with the multipactor electrons plays a relatively minor role. When saturation occurs, the secondary emission coefficient is unity, corresponding to the "first cross-over point" in the secondary electron yield curve. The parameters attained in the steady state agree with the predicted values from an analytic theory. The analysis is extended to include the effects of an external magnetic field.

Multipactor is a well known phenomenon of $\mathrm{rf}$ breakdown in microwave cavities, windows, satellite $\mathrm{rf}$ payloads, and accelerator structures [1-3]. When an AC electric field exists across a gap, an electron from one surface is accelerated toward the other surface, the impact upon which may release more than one electron by secondary emission. It is easy to see that if the electron transit time across the gap equals to half of the rf period, a resonant discharge could result.
\end{abstract}

There exist few theoretical analyses of multipactor, most of which are concentrated on the response of a single electron to an imposed rf electric field. Analytic expressions have been derived for the phase of the emitted electron, and the range of the rf electric field in which a stable, steady state multipactor may exist $[1,4]$. While some calculations have included the space charge effects associated with the multipactor electrons [4,5], most of these calculations omit the important processes of loading and detuning of the rf cavities as the multipactor current grows [2]. In this paper, we use a simple model to address these issues, the analysis of which yields interesting information on the multipactor saturation level, the saturation mechanism, the time scale over which

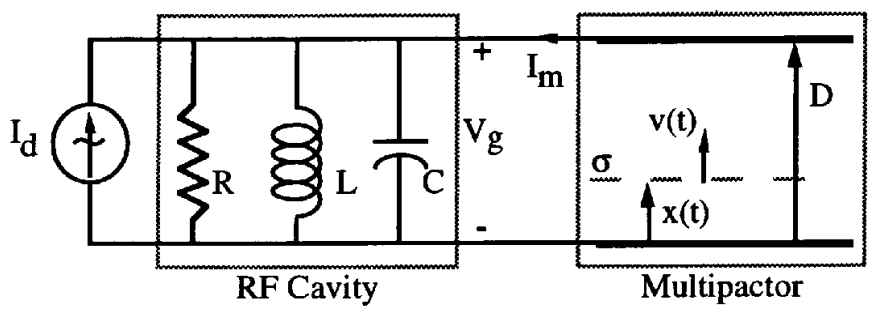

Fig. 1: Model of interaction between rf cavity and multipactor discharge. multipactor evolves, and possibly the drastic transient growth of multipactor current before the steady state solution is reached.

For simplicity, we shall use a one dimensional model where the multipactor occurs inside a planar gap [Fig. 1]. The gap separation is $D$ and the gap voltage is $V_{g}(t)$. The multipactor discharge is modeled by a single electron sheet of surface density $\sigma$ that moves across this gap. Upon impact on a gap surface, a new electron sheet is generated by secondary emission. We assume that the voltage $\mathrm{V}_{\mathrm{g}}$ that drives the multipactor is provided by an rf cavity, of characteristic frequency $\omega_{0}$ and quality factor Q [Fig. 1]. As the multipactor electron sheet moves inside the gap, it induces a wall current, $I_{m}(t)$, which loads the cavity. Thus, the present model allows for the progressive loading and detuning of the cavity as the multipactor current builds up. This loading, in turn, modifies the electron's energy and phase at impact.

Hereafter, we shall use dimensionless quantities with the following normalization scales: D for distance, $\omega_{0}$ for frequency, $1 / \omega_{0}$ for time, $v=\omega_{0} D$ for velocity, $U=m v^{2}$ for energy, U/e for voltage, $\mathrm{E}=\mathrm{U} / \mathrm{eD}$ for electric field, $\Sigma=\varepsilon_{0} \mathrm{E}$ for surface charge density, $A \Sigma v / D$ for current. Here, $m$ is the electron mass, $\mathrm{e}=1.602 \times 10^{-19}$ Coulomb, $\mathrm{A}$ is the surface area of the gap, and $\varepsilon_{\mathrm{o}}$ is the free space permittivity. The cavity is driven by the normalized ideal current source $\mathrm{I}_{\mathrm{d}}$, and by the multipactor current $\mathrm{I}_{\mathrm{m}}$, according to the circuit equation [Fig. 1]:

$$
\left(\frac{d^{2}}{d t^{2}}+\frac{1}{Q} \frac{d}{d t}+1\right) V_{g}(t)=\frac{d}{d t}\left[I_{d o} \sin (\omega t+\phi)+I_{m}(t)\right]
$$

Here $\mathrm{I}_{\mathrm{do}}$ is the amplitude of the driver current of normalized frequency $\omega$, and $\phi$ is the phase at time $t=0$. We set $\omega=1$ in this paper (i.e., resonantly driven). The normalized multipactor current $\mathrm{I}_{\mathrm{m}}$ is induced by the electron sheet motion:

$$
I_{m}(t)=-\sigma(t) \bullet \frac{d}{d t} x(t),
$$

where $\sigma$ is always positive, by convention. It is this term that is solely responsible for the non-linear beam loading and frequency detuning of the cavity by the multipactor, as readily seen from Eq. (1) and Fig. 1.

The force law for the electron sheet is

$$
\frac{d^{2} x}{d t^{2}}+\omega_{C}^{2}\left(x-x_{o}\right)=V_{g}+\sigma \bullet\left(x-\frac{1}{2}\right),
$$

where the initial plate of origin is denoted by $x_{0}=0$ or 1 . The first term on the right hand side represents the force due to the gap voltage and the second term the force due to the image charge (of the multipacting electron sheet) on the plates. We 
have included a general transverse magnetic field with a cyclotron frequency $\omega_{\mathrm{C}}$, normalized to the driver frequency $\omega$.

On impact with a plate at time $t_{i}$, the incident electron sheet is removed and a new sheet of surface charge is released by secondary emission. The post-impact surface charge density $\sigma\left(t_{i}^{+}\right)$is related to the pre-impact charge density $\sigma\left(t_{i}^{-}\right)$by

$$
\sigma\left(t_{i}^{+}\right)=\delta \bullet \sigma\left(t_{i}^{-}\right),
$$

where $\delta$ is the coefficient of secondary emission [6] which depends on the electron impact energy, $E_{i}$ [7]. Here, $E_{i}=(d x / d t)^{2} / 2$, evaluated at $t=t_{i}^{-}$. In addition, we adopt Vaughan's empirical formula [6] for $\delta$. This function is shown in Fig. 2, where $\delta_{\max }$ is the maximum value of $\delta$, occurring at an impact energy, $\mathrm{E}_{\max }$. Fig. 2 shows that $\delta=1$ at two values of impact energies, $E_{1}$ and $E_{2}$. The lower energy $E_{1}$ is designated as the "first cross-over point".

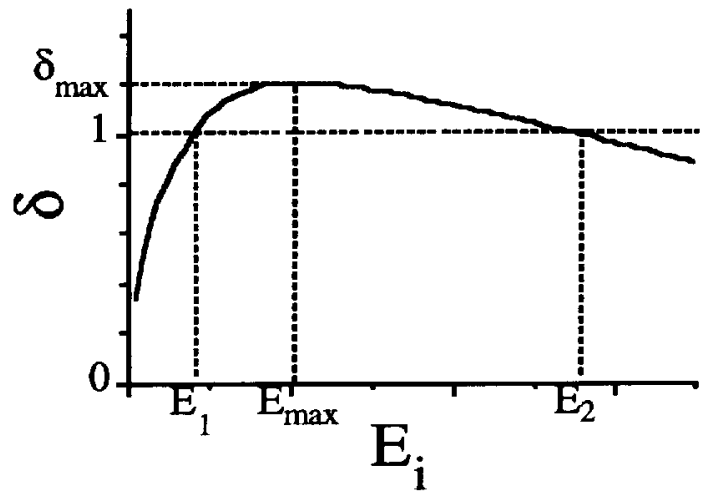

Fig. 2: Secondary electron yield, $\delta$, as a function of impact energy $\mathrm{E}_{\mathrm{i}}$.

In the present formulation, multipactor affects its own evolution in two ways. First, it loads the cavity and changes the gap voltage; this process is entirely accounted for by the term $I_{m}$ in the circuit equation (1). We may designate this effect as the "beam-loading effect". Second, as the multipactor current builds up, the space charge force may become appreciable, and may influence the arrival phase of the impacting electron relative to the $\mathrm{rf}$ phase. This second effect is modeled by the last term in the force law, Eq. (3), and may be called the "space charge effect".

We have established analytically the necessary conditions for the existence of steady state solutions according to Eqs. (1) - (5). One condition for a steady state is that, every cycle, the sheet has to arrive at the same plate when the phase of the voltage is the same (a "fixed-phase" [4]). Otherwise, the sheet will keep drifting in phase, and may eventually hit one of the plates in the wrong phase of the rf cycle, quenching the multipactor. This phase stability is discussed elsewhere [1, 4]. The second condition is that steady-state has to occur when $\delta=1$, resulting in a constant $\sigma$ in Eqs. (2) and (3) above. This occurs for two impact energies, but a simple physical argument shows that only the first cross-over point $\mathrm{E}_{1}$ in Fig. 2 gives the stable steady state solution [8]. Hence this condition also fixes the impact energy to $E_{1}$ in the steady state.
To obtain the steady state solution, we solved Eqs. (1), (2), and (3) with a constant $\sigma$. Then, we used the fixed phase condition and an impact energy of $\mathrm{E}_{1}$ to relate the initial values of $\mathrm{V}_{\mathrm{g}}$ and its derivative in Eq. (1) to their final values. This results in the steady state values of the charge density, the gap voltage amplitude, and its value at impact. The numerical results, to be discussed below, agree quite well with the steady state predictions of the analytic theory.

To study transient evolution, we assume that the driver current $I_{d}$ has been turned on for all time so that the cavity is already filled with $\mathrm{rf}$ for $\mathrm{t}<0$. The multipactor current is "turned on" at $\mathrm{t}=0$, in the form of an electron sheet with initial surface charge density $\sigma_{o}$ that is released from the plate $x=0$, with zero velocity [9]. The initial phase $\phi[$ Eq. (1)] at which $\sigma_{o}$ is launched is chosen so that this initial electron sheet strikes the other plate in about half an rf cycle. In most cases we run, the precise values of these initial data are not critical. Our simulations thus far have been restricted to twosurface, first-order multipactor [1], i.e., an electron released from one surface always strikes the other surface without momentarily stopping within the gap. The major free parameters are: $\mathrm{Q}$ and $\mathrm{I}_{\mathrm{do}}$, after having fixed $\delta_{\max }=1.2, \mathrm{E}_{\max }=$ 0.36 , and $\omega=1$. [In dimensional units, if the rf cavity has a natural frequency of $1 \mathrm{GHz}$, and a gap separation of $0.22 \mathrm{~cm}$, over which multipactor occurs, these parameters correspond to an ideal rf driver current exactly at $1 \mathrm{GHz}$, and $\delta$ reaches a maximum value of 1.2 when the impact energy is $400 \mathrm{eV}$.]

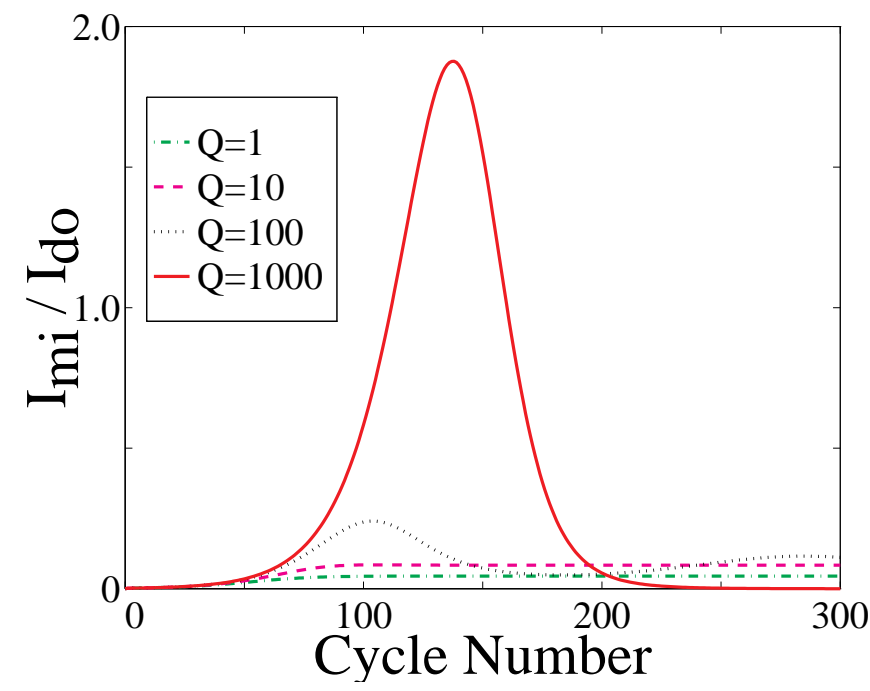

Fig. 3: Transient evolution of multipactor current at impact, in units of the drive current amplitude $I_{d o}$, for various values of quality factor, $\mathrm{Q}$.

Shown in Fig. 3 is the multipactor current, monitored at impact, in units of the driver current amplitude $I_{d o}$, for $Q=1$, $10,100,1000$. The very low value of $\mathrm{Q}$, e.g., $\mathrm{Q}=1$, is included in our study to show the trend of multipactor in a non-resonant structure -- one that is relatively immune to beam loading, such as a window. It is seen from Fig. 3 that in a high $\mathrm{Q}$ cavity, the multipactor current may reach a very high level in a transient manner before it settles down to steady state. Throughout the transient development of the 
multipactor, the peak rf gap voltage in each cycle changes relatively little, for either the $\mathrm{Q}=1$ case or the $\mathrm{Q}=1000$ case [Fig. 4]. The secondary emission coefficient $\delta$ also stays around unity, specifically in the vicinity of the first cross-over point $\left(E_{1}\right)$ in Fig. 2, as discussed earlier. Our numerical results show that the impact energy indeed approaches $E_{1}$ asymptotically in time. When the steady state is reached, the gap voltage at impact, therefore, is insensitive to $\mathrm{Q}$, as shown in Fig. 4. We have spot-checked that the steady state values of the gap voltage, of the electron impact phase in the rf cycle, and of the surface charge density, are all in good agreement with those obtained from our analytic formulation.

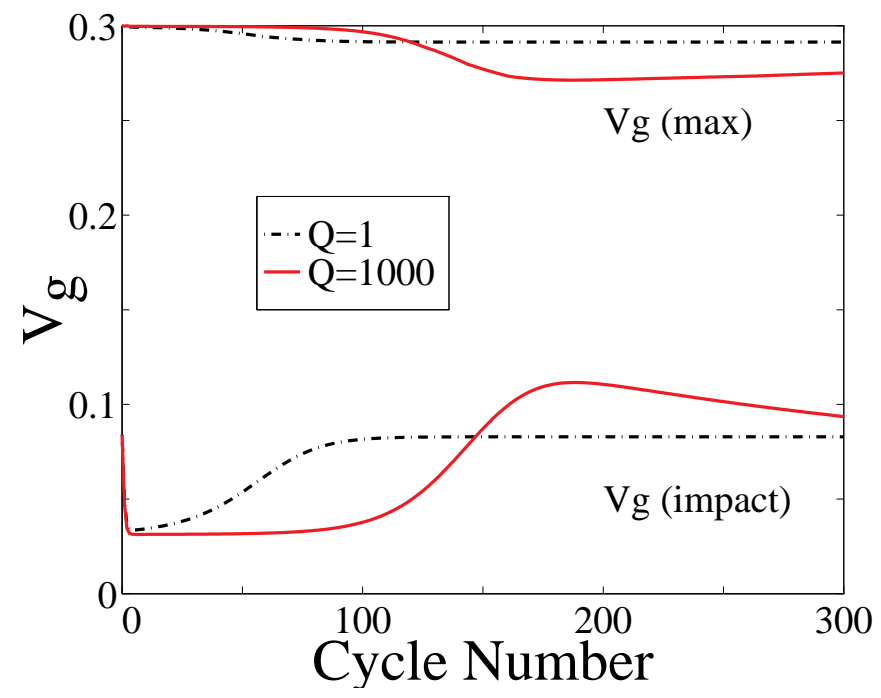

Fig. 4: Evolution of the peak gap voltage, $\operatorname{Vg}(\max )$, and of the gap voltage at the instant of electron impact, $\mathrm{Vg}$ (impact), for $\mathrm{Q}=1$ and $\mathrm{Q}=1000$.

The numerical results show that, as long as $\mathrm{Q} \sim 10$, the beam loading effect is far more important than the space charge effect in determining the saturation level of the multipactor current. The disparity of their relative importance becomes increasingly more pronounced as $\mathrm{Q}$ increases, as high Q cavities can be more readily detuned by a multipactor current. This also explains the sensitivity in the high $\mathrm{Q}$ cavities . The fraction of rf power dissipated in the RLC circuit,

$\left\langle-V_{g}\left(I_{d}+I_{m}\right)\right\rangle /\left\langle V_{g} I_{d}\right\rangle$, is shown in Fig. 5. Here < $\rangle$ denotes the average over the transit time of an electron. The rf energy stored in a high Q cavity is capable of driving the multipactor current to a large amplitude when the condition becomes favorable. This gives the tantalizing clue that, in reality, the large amount of energy stored in high Q cavities may relax via a multipactor discharge, albeit transiently in time, and locally in space. In an example, we show that as much as $20 \%$ of the energy stored in a high Q cavity may be discharged in a single overshoot of the multipactor!

We have included the effect of a non-zero transverse magnetic field for the $Q=1000$ case [7]. The presence of the magnetic field is found to reduce the strength of multipactor, according to this (much) simplified model. Specifically, when the transverse magnetic field yields a cyclotron frequency equal to 0.3 times the drive frequency, the peak multipactor current in the transient evolution is approximately $1 / 8$ of that in the case of zero magnetic field.

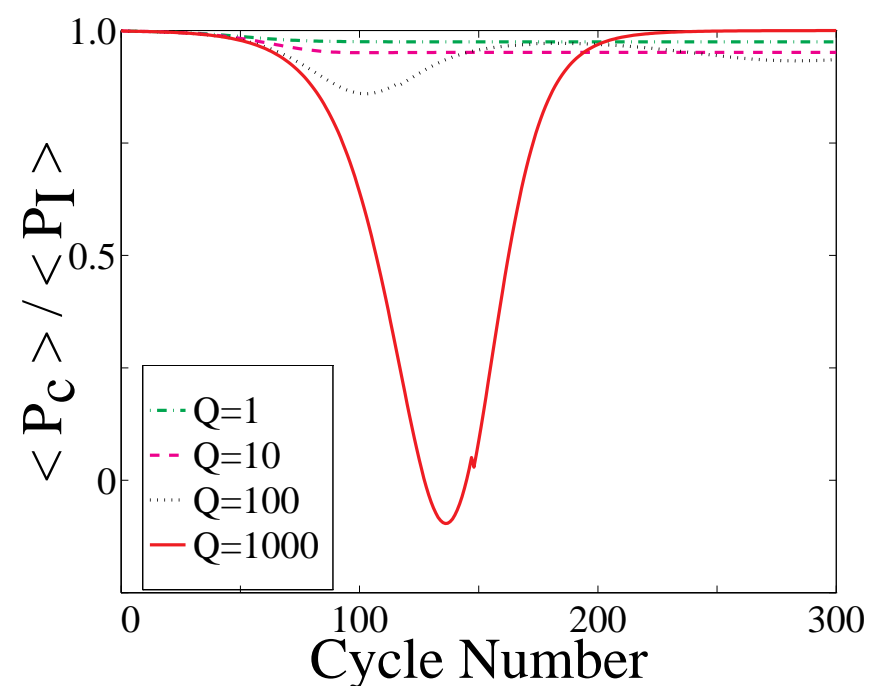

Fig. 5: Time history of the power consumed by RLC circuit, in units of the input power, at various values of $Q$.

We have benefited from many useful conversations with David Chernin, Spilios Riyopoulos, Perry Wilson, Jake Haimson, and Richard Briggs. This work was supported by NRL/ONR.

\section{References}

[1] J. R. M. Vaughan, IEEE Trans. ED-35, 1172 (1988).

[2] A. S. Gilmore, Microwave Tubes, (Artech House, Norwood, MA), p. 474 (1986).

[3] G. A. Loew and J. W. Wang, SLAC Pub. No. 4647 (1988); A. D. Woode and J. Petit, Microwave Journal, (January, 1992), p. 142.

[4] S. Riyopoulos, D. Chernin, and D. Dialetis, to be published.

[5] F. M. Mako and W. Peter, Proc. 1993 IEEE Part. Acc. Conf., p. 2702 (1993).

[6] J. R. M. Vaughan, IEEE Trans. ED-36, 1963 (1989); A. Shih and C. Hor, IEEE Trans. ED-40, 824 (1993).

[7] In this paper, we ignore the dependence of $\delta$ on the angle of impact, even when an external magnetic field is present. We assume that $\delta$ depends only on the total impact energy.

[8] If the impact energy $E_{i}$ is greater (less) than the first cross-over point $\mathrm{E}_{\mathrm{i}}$, more (fewer) electrons will be released by secondary emission. As a result, more (less) energy will be drained from the cavity as it accelerates more (fewer) secondary electrons. This leads to a lower (higher) gap voltage which makes $E_{i}$ closer to $E_{1}$ in subsequent cycles. Thus $E_{1}$ is a stable steady state solution. A similar argument shows that the second cross-over point, $\mathrm{E}_{2}$ in Fig. 2, is unstable.

[9] It is shown by Riyopoulos et al [Ref. 4] that the inclusion of the initial velocity of the secondary electrons does not qualitatively change their steady state solutions. 